\title{
A qualitative exploration of the experience and quality of life of patients, and their family carers, admitted to a specialist eye hospital with microbial keratitis
}

\section{Shelley Anne Tranter}

Sydney Hospital and Sydney Eye Hospital

\section{Maria Careras-Aguas \\ University of Sydney}

Mandy Riddell

Sydney Hospital and Sydney Eye Hospital

Joanna McCulloch

Sydney Hospital and Sydney Eye Hospital

Therese Riley

Sydney Hospital and Sydney Eye Hospital

\section{Stephanie Watson}

University of Sydney

Lin Perry ( $\boldsymbol{\sigma}$ Lin.Perry@uts.edu.au )

University of Technology Sydney https://orcid.org/0000-0002-8507-1283

Research article

Keywords: Microbial keratitis, patient experience, carer, hospitalisation, quality of life, nursing

Posted Date: April 12th, 2019

DOI: https://doi.org/10.21203/rs.2.9130/v1

License: (c) (1) This work is licensed under a Creative Commons Attribution 4.0 International License. Read Full License 


\section{Abstract}

Background: Microbial keratitis is a vision-threatening condition requiring intensive treatment. Understanding patients' and carers' experience during and after hospitalisation can inform improvements in care and outcomes. This study explored the vision-related quality of life, and the experiences of patients with microbial keratitis and their family carers when admitted to a quaternary referral eye hospital in Australia Methods: The study employed mixed methods, including qualitative interviews and a survey in hospital and a telephone interview post-discharge. A convenience sample was recruited of 33 patients with microbial keratitis who presented to hospital between March and October 2017, and 10 of their family carers. Semi-structured interviews were audiotaped, transcribed verbatim, coded and analysed using thematic analysis. Patient participants completed the National Eye Institute Visual Functioning Questionnaire - 25 (NEIVFQ-25). Results: Qualitative analyses identified two main themes: Saving sight, with subthemes of costs of saving sight, and travel and transportation; Safe-guarding home and normal life with subthemes of family, work and pastimes. A group mean NEIVFQ-25 score of 74 was similar to other ophthalmic disease groups but pain scores were higher. Conclusion: Findings provide insights into the experiences and often unspoken concerns of microbial keratitis patients and their family carers, revealing the priority and the associated costs of saving sight, and the implications for family and lifestyles. These patients reported similarly reduced vision-related quality of life but greater pain compared to other ophthalmic groups. Findings point to ways to improve their experience and potentially reduce the high rates of unplanned representations of this patient group.

\section{Background}

Microbial keratitis is a commonly occurring ocular emergency of sudden onset; in 2010 it resulted in over 58,000 presentations to Emergency Departments in the United States (1). Those affected can suffer significant pain, distress (2) and scarring, with reduced quality of life (3). It is a common cause of visual impairment in working age adults in Australia (4), incurring heavy costs for individuals, society and the health system ADDIN EN.CITE $(5,6)$.

Microbial keratitis is an important cause of hospital admissions, especially among older people, those with severe disease, unable to self-administer topical therapy (7) or living distant from the centres capable of providing the specialist treatment this condition requires (8). A prolonged hospital stay may result, which can significantly impact patients and families (9), especially where home is a long distance from the hospital. In Australia average lengths of stay for this condition are reducing, but remain significant. In 1998-2002, patients aged over 60 years with microbial keratitis admitted to an Australian specialist eye hospital had an average length of hospital stay of 17.6 days, and $50 \%$ experienced one or more complications (7). A decade later (2013-14), similar patients admitted to the same hospital had a mean length of hospital stay of 11.4 days, with only one in ten experiencing complications; one in twenty required emergency readmission (10). 
Few studies examine the experiences of ophthalmic patients, or do more than visual testing. Visionrelated quality of life has been examined in various vision-impaired populations using the National Eye Institute Visual Functioning Questionnaire - 25 (NEIVFQ-25) (11, 12), but the experiences of patients with microbial keratitis, and their family carers, are not reported.

The aim of this study was therefore to explore the experiences of patients with microbial keratitis admitted to a quaternary referral eye hospital in Australia, and that of their family carers, and to examine patients' vision-related quality of life.

\section{Methods}

\section{Design}

This was a mixed methods study combining qualitative and quantitative elements (13). The major component entailed an exploratory, descriptive study using narrative methods to collect rich and detailed data (14). The quantitative component entailed completion of a validated questionnaire by patient participants only, measuring their vision-related quality of life.

\section{Participants and setting}

The study site was a quaternary referral hospital for eye diseases in metropolitan Australia which admits approximately 200 patients per year with this condition (15). Patients were eligible to participate if they were admitted with a clinical diagnosis of microbial keratitis, were 18 years of age and over, medically stable, able to converse in English, and had no documented cognitive impairment. Their carer or family member (someone identified as a non-professional carer or support person of a recruited patient) was also invited to participate. Recruitment occurred between March and October 2017 and concluded with data saturation, when no new information emerged during interviews.

\section{Exploration of the patient and family carer experience}

Semi-structured interviews allowed participants to determine what aspects of their experience were discussed, and to provide depth and detail. The interview questions were developed by the research team, pilot tested and refined with the first participants. The initial interview questions focussed on the patients' journey, from their impetus to seek medical attention to their hospital experiences during admission for the treatment of keratitis. Follow up interviews with patients after discharge were conducted by telephone and focused on the experience of hospital discharge and the period following their return home.

Consenting patients and their family carers were interviewed by the research officer, a Registered Nurse with no clinical role in the hospital. Patients and family carers were interviewed separately to enable free and open discussion. Initial interviews were conducted in a quiet room on the hospital ward during the inpatient stay, or (for some carers), by telephone. Follow-up patient interviews were conducted by telephone approximately one to two weeks after discharge from hospital. Interviews were digitally audiorecorded and transcribed verbatim using a professional service. 


\section{Quality of Life assessments}

At conclusion of the narrative component of the initial patient interviews, the research officer verbally delivered the National Eye Institute Visual Functioning Questionnaire - 25 (NEIVFQ-25).

The NEIVFQ-25 is a validated short-form version of the 51-item NEIVFQ (12), comprising one general health question and 24 questions measuring eleven domains of self-reported vision-targeted health status summed into a total score. The questions are variously structured; most are scored on a 5 or 6 point scale ranging from 1 "not affected at all", to 4 "severely affected" or recording activity ceased due to eyesight or other reasons. True - false responses are scored on a 5-point scale from 1 "definitely true" to 5 "definitely false". All responses are converted to a score between 0 and 100 with higher scores representing better vision-related quality of life. An overall score is calculated as the mean of domain scores (12).

The NEIVFQ-25 has undergone psychometric evaluation and been used with patients with ocular conditions including microbial keratitis, and the general population. Tests of validity and reliability have demonstrated acceptable values, with more than $85 \%$ of the variance of long-form scores demonstrated by this ( 25 -item) short-form version. Internal consistency estimates (Cronbach alpha values) range from 0.71 to $0.85(16)$.

\section{Data analysis}

Using thematic analysis, transcripts were coded, patterns of meaning sought and themes mapped, revised, defined and named (17) using NVivo Pro $11^{\mathrm{T}}$ software. The research officer conducted preliminary analysis, sharing the coding with research team members (18). Team members sampled transcripts and independently coded; codes and themes were discussed and agreed.

Data from the NEIVFQ-25 survey were transformed in line with the developers' instructions using SPSS for Windows Version 23 (19). Descriptive statistics presented participant demographic data, length of hospital stay and instrument domain and total scores.

The NEIVFQ-25 total and domain scores were integrated with qualitative findings to locate this sample in relation to other ophthalmic groups, to assist understanding the impact of microbial keratitis, visual function and hospital admission in relation to patients' experience and quality of life.

\section{Research rigor}

To reduce potential bias or a perceived conflict of interest the interviewer was external to the ophthalmology service and took a neutral stance to service-related experiences narrated by participants. Trustworthiness was enhanced by detailed description of processes and participants, with interview quotations supporting analysis interpretations and credibility. The research team (clinicians and academics internal and external to the research site) met regularly to discuss and review data collection, coding and analysis (18). Follow up interviews offered patients an opportunity to confirm a summary of 
the main points from initial interviews. The NEIVFQ-25 has been evaluated extensively $(11,12)$ and shown to perform well.

\section{Ethical considerations}

Approval to conduct the study was obtained from South Eastern Sydney Human Research Ethics Committee (reference: 151246; HREC/15/PoWH/491). A suitably trained professional was available to provide support for any distress that may have arisen from discussion of potentially sensitive topics (20).

\section{Results}

Data saturation was evident at recruitment of 33 patients. During the study period 97 patients were admitted with a diagnosis of microbial keratitis; seven were non-English-speaking, four declined to participate, seventeen were unavailable for interview at suitable times, mostly due to limited research officer availability, and thirty six patients with severe ocular pain, cognitive impairment or complex medical conditions were ineligible. Initial interviews with patient participants were recorded at mean (SD) 21.7 (8.6) minutes duration. Nine participants received follow-up phone interviews between one and two weeks after hospital discharge, averaging 10.2 (4.2) minutes duration. Ten family carers were recruited and interviewed, averaging 19.0 (6.4) minutes duration.

\section{Participant demographic details}

Patient participants had an average age of 63.0 (SD 20.7; range 23.0 to 96.0 ) years and $64 \%$ were male. The median duration of their hospital admission at the time of interview was five days, with median (25, 75 quartile) total in-patient duration of $10.0(5.8,20.6)$ days, ranging 2 to 119 days. The majority ( $\mathrm{n}=21$, $64 \%)$ lived in major cities and towns; for two-thirds of the sample $(n=14 ; 42 \%)$ this was the same city as the research hospital. Two participants resided in other states (Table 1).

Family carer participants had an average age of 64.1 (SD 10.2; range 40 to 75) years, and were predominantly (90\%) female. Six were patients' wives, one was the patient's husband and one each was described as a patient's partner, niece or daughter.

Table 1 Demographic characteristics of patient and family carer participants 


\begin{tabular}{lll} 
Characteristics & Patients $(\mathbf{n = 3 3 )}$ & Carers $(\mathbf{n = 1 0})$ \\
\hline Age (years) mean (SD) & $63(20.7)$ & $64(10.2)$ \\
\hline Gender n (\%) Male & $21(64)$ & $1(10)$ \\
\hline Female & $12(36)$ & $9(90)$ \\
\hline Australian-born n (\%) & $22(67)$ & $5(50)$ \\
\hline Living in NSW n (\%) & $31(94)$ & $9(90)$ \\
\hline ASGC-RA category n(\%) & $21(64)$ & $6(60)$ \\
Major city & & \\
\hline Inner regional & $7(21)$ & $3(30)$ \\
\hline Outer regional & $5(15)$ & $1(10)$
\end{tabular}

SD = standard deviation, NSW = New South Wales, ASGC-RA = Australian Standard Geographical Classification - Remoteness Areas

\section{Interview themes}

Analysis of the patient interviews revealed two major themes. Saving sight had subthemes of costs of saving sight, travel and transportation, and unpreparedness; Safe-guarding home and normal life centred around family, work and pastimes. Themes were common findings across patients and their family carers. In the text, patients and carers are identified by numbers prefixed with $\mathrm{P}$ and $\mathrm{C}$, respectively

\section{Theme 1: Saving sight}

All patients commented on the primary importance to them of maintaining or restoring their eyesight. This was their focus, pre-occupying their thoughts:

... I think this looks like it's the last resort, you know. I had been worrying that I might have...lost my eye. (P7)

The priority of saving sight was also illustrated by carers, with one adamant that:

He [her husband] would rather lose a leg than his eyesight. (C8)

The possible effect of the disease on vision was a source of deep anxiety and worry. An immediate consequence was that it was crucial that treatment continue, even when things were not going as well as hoped. Participants clung to hope; importantly, they wanted their doctors to do the same:

That's my big, big, deep worry, which I'm being very honest and opening up about now, but I'm pushing that down, thinking that is my worst worry, I might lose the eye. (P5) 
When asked if the patient had discussed this with the medical team, no-one responded affirmatively. At this relatively early stage of treatment, a pervasive attitude of non-disclosure was revealed, founded in the fear that medical staff might terminate treatment they regarded as potentially curative:

No, I haven't. I don't want to discourage them. (P5)

A sub-theme expounded the cost of saving sight, reflected in a number of ways. Patients would endure the intensive treatment, in the form of eye drop regimens and injections, if it meant that they could continue to hope their eyesight may improve or that their eye/s could be saved. Eye drops were administered hourly, often day and night, or even more regularly for a number of days before reducing the frequency. Such regimens were exhausting but the participants accepted this without demur as it was required to treat their keratitis and it signalled to patients that hope was not lost. Patients appreciated that such regimes were also challenging for nurses to deliver, because of the workload but also because of the toll on an empathic nurse to have to wake someone hourly:

On the hour every hour .. the poor nurse has got to come and wake, and they say, oh, sorry, I've got to wake you. But it's no good them being sorry, it's... part of the job and part of the... that is the treatment. (P11)

The treatment imposed considerable strain, and the nightly regimen resulted in sleep deprivation. I could sleep on a barbed-wire fence......it's not normal, I don't normally sleep in the daytime, and I don't have naps. (P11)

Nevertheless, whatever the problems or discomfort associated with their admission to hospital and their treatment, the facts of this were not per se a source of worry as they were recognised as a necessity to be endured:

.... because I accept that it has to be done. It is the best thing for me. ... they are all working for the same cause I am working for which is getting it right. (P2)

The condition itself caused pain, but patients also had to endure discomfort ranging to severe pain from intraocular injections. Patients' responses were often, but not invariably, stoic, initially at least:

I wasn't really worried about it but it turns out he gave me five needles in the eye which didn't impress me one bit. (P3)

There was comfort in knowing that they or their loved one were in a hospital where the staff were experts in eye care. The 'expert' status of the staff of a specialist eye hospital was a source of reassurance for patients and families:

I was just grateful to be under a roof where I knew somebody was handy to look at it [the eye], and they all knew what they were doing. (P5) 
I couldn't do anything for him at home because he was practically crying from the pain and I know he was in the best place getting the best care. (C3)

A second sub-theme addressed travel and transportation. Travel to and from the hospital was mentioned by all participants. Most patients had relied on a carer to drive them and, with less than half $(42 \%)$ living in the city where the hospital was located, for many this entailed long distances. For some, the journey to the hospital had involved a number of steps, and use of public transport or flights from regional areas, other states or territories. Some carer participants, especially those who lived in regional or rural areas, were not confident in driving to or across the city. Even those who did drive could find it difficult to locate the hospital and appropriate parking.

I would not even attempt to drive into the city. I wouldn't have a clue (C9)

Transportation continued to be a concern after they arrived at the hospital, with anxieties about getting home from hospital. Navigating an unfamiliar environment with a newly-discharged patient could appear daunting. One patient had travelled to the hospital from a regional area in an ambulance and was concerned about how to return home:

She just said oh no you'll just have to get a train home. And I'm like I can't see to get the trains home. (P30)

This participant was asked at follow up how her trip home had gone:

... it was really scary, really hectic, and I was knocked over while on the escalators. (P30)

The third sub-theme focused on unpreparedness. For all patients the suddenness of symptom onset meant they were unprepared: planning and preparation was rushed, incomplete and/ or inadequate.

Went home, didn't pack it, no we didn't. Yes, we did. We went home, swapped cars around, used the wife's car instead of mine. (P 27)

A number of the patients remained unprepared for hospital admission even at the point of admission: they had attended the hospital unaware they might be admitted:

... all they said was you're going to get some eye drops. I'm like, some really strong eye drops, okay. The doctors here said, oh, you're staying. I'm like, what? (P26)

Unexpected hospital admissions, and unexpectedly protracted length of stay, could be accepted as a necessary imposition for the sake of saving their sight but unpreparedness resulted in patients not having the clothes or other essential items with them. One patient was unable to source suitable incontinence pads or his usual medication:

I came in expecting to be here for three days, and I'm still in. I'll be here probably eight, nine days by the time I leave, so I run out of things. (P24) 
The journey, the consequent admission and treatment and then the processes of hospital discharge and return home entailed a great deal of anxiety and upset for most participants. However, these were necessary processes and it was recognised that these were the costs of gaining expert treatment for their eye condition and hopefully saving sight.

\section{Theme 2: Safeguarding home and normal life}

This theme centred around family, work and hobbies; throughout, the dominant sense was one of anxiety and concern at the implications of the current situation, with a desire to preserve normal life.

Some patient participants were more concerned for their family, especially their partner, than for themselves. In three instances the spouse at home was not well and the patient was their carer, compounding the stress of these patient participants.

Yes, because, yes, she does get sort of tired and out of breath and other things. So, I said to the nurse I tried to ring the wife (P16)

Family carer participants were happy in the knowledge that their loved ones were receiving expert care but were nonetheless concerned about them and for what the future might hold for them. Some patient participants already had some experience of declining sight, were only too aware of the potential for acute deterioration and what the implications of this might be:

Well he gets a bit cranky. I think that is because he gets frustrated because there are a lot of things he used to do and he can't do any more. He can't see properly. Even with a screw he tries and I say I will do it and he says no. In the end I have to do it because he can't see and it's like he knows he can't see but he doesn't want to believe it. (C5)

Spouses and family members found themselves with role reversal, and were often placed in new and unfamiliar roles, such as becoming the main carer for a child or the farm manager during the patient's admission. The unfamiliarity of this was an additional source of anxiety for both parties. This could be further compounded by patients' separation and isolation from their families; patient participants reported missing loved ones. One patient, whose home was 12 hours by road from the hospital and who had been hospitalised over a week when interviewed, reported missing the grandchildren she usually saw every day.

Most patients did not work but for those who did there were work concerns. They worried how they would manage, and how their work was continuing while they were in hospital. In some instances patient participants continued to at least try to work remotely during their hospital admission:

I'm sort of running... well, not running, but I'm working on quite a big project which I'm sort of quite in the middle of, ... they're sort of struggling a bit without me there, so I'm missing out on a fair bit of work.... (P25) 
Further, there were concerns regarding their finances whilst on sick leave, and anxieties that long term hospitalisation and regular follow up would drain future finances, especially for those who lived long distances from the hospital:

... That little nest egg will be gone. But still, hopefully at the end of August I'll still have an eye. (P8)

Patients and family carers were uneasy about the outcome of the infection and the admission on eventual vision, and for the effect of vision for future employability. This included future ability to continue working and being able to maintain a driver's licence and hence mobility.

Probably my biggest concern is whether I will be okay to drive. (P17)

For those who did drive, the ability to continue driving was very important.

For patient participants who were retired, a major concern centred on their ability to maintain activities, hobbies and interests. For one patient, belonging to the men's shed and doing woodwork was extremely important but this was hard for him to reconcile with the risk of eye damage from dust and wood particles:

Only for the club [men's shed] I think I would be in me grave now. I'm not going anywhere near the club until me eye is right. (P7)

One participant could only see the future in terms of losses, and lamented all the changes that would occur once he went home:

.... I used to always do the gardening. I've liked to paint houses, I liked to do physical things like carpentry and wash the car, and I can't do any of that anymore. (P18)

\section{Self-reported vision-related quality of life: NEI VFQ-25 results}

With total and domain scores (Table 2) calculated of a maximum possible score of 100, a mean (SD) NEIVFQ 25 score of 74 (21.9) was reported. Participants' median score of 83.9 indicated a right skewed pattern, with more patients reporting above-average than below-average scores. Three participants reported scores below the 25 th centile, indicating severely depressed vision-related quality of life. Selfreported health revealed a similarly right-skewed pattern; a relatively low mean of 54.6, with a much higher median score of 75 .

Of the domain scores, general vision was particularly affected, with the lowest average score. By contrast, colour and peripheral vision were rated as virtually unaffected. Ocular pain scores ranged from very low to maximal, with substantial variation revealed by a SD of 21.5 (Table 2).

Table 2: National Eye Institute Visual Functioning Questionnaire-25 total and domain scores 


\begin{tabular}{llllllll} 
& N & Min. & Max. & Mean & SD & Median & 25,75 Q \\
\hline General health & 33 & 0 & 100 & 54.6 & 26.1 & 75.0 & $37.5,87.5$ \\
\hline Vision specific: mental health & 33 & 12.5 & 100 & 67.0 & 24.3 & 81.2 & $53.1,90.5$ \\
\hline Vision specific: role difficulties & 33 & 0 & 100 & 61.4 & 30.3 & 75.0 & $62.5,87.5$ \\
\hline Vision specific: dependency & 33 & 0 & 100 & 67.9 & 27.2 & 91.7 & $75.0,91.7$ \\
\hline Driving & 25 & 0 & 100 & 65.9 & 41.4 & 91.7 & $37.5,100.0$ \\
\hline General vision & 33 & 0 & 80 & 50.9 & 20.1 & 60.0 & $40.0,80.0$ \\
\hline Colour vision & 32 & 25 & 100 & 90.6 & 23.5 & 100.0 & $100.0,100.0$ \\
\hline Peripheral vision & 33 & 25 & 100 & 73.5 & 27.9 & 100.0 & $62.5,100.0$ \\
\hline Near activities & 29 & 0 & 100 & 63.2 & 28.6 & 83.3 & $37.5,100.0$ \\
\hline Distance activities & 21 & 0 & 100 & 65.5 & 32.2 & 91.7 & $66.7,100.0$ \\
\hline Vision specific: social functioning & 26 & 12.5 & 100 & 78.4 & 30.1 & 100.0 & $100.0,100.0$ \\
\hline Ocular pain & 33 & 12.5 & 100 & 62.1 & 21.5 & 62.5 & $50.0,75.0$ \\
\hline TOTAL NEIVSF-25 & $\mathbf{1 3}$ & $\mathbf{2 1 . 3}$ & $\mathbf{9 3 . 4}$ & $\mathbf{7 4 . 0}$ & $\mathbf{2 1 . 9}$ & $\mathbf{8 3 . 9}$ & $\mathbf{5 6 . 5 , 9 2 . 0}$
\end{tabular}

\section{Discussion}

Our study reports the experience of microbial keratitis and admission to hospital for its treatment in patients and their family carers, and the resultant poor vision-related quality of life. Patient and carer experiences centred around themes of Saving Sight and Safe-guarding home and normal life. Patients' vision-related quality of life scores demonstrate similarly depressed vision-related quality of life but greater pain compared to other ophthalmic diagnostic groups.

\section{Saving sight}

Our study highlights the importance for people of maintaining good sight; this aligns with findings of a study conducted in Australia by Vision 2020 (21). The majority of people surveyed reported going blind as worse than a heart attack or losing a limb. Sight was the most valued sense, particularly for those aged 65 and older.

Our sample patients were prepared to tolerate substantial pain and privations in silence to retain hope of saving their sight. Similarly, patients requiring intra-vitreal injections for age related macular degeneration reported experiencing financial and travel burdens and treatment related anxiety but were willing to accept this in order to preserve their precious sight (22). Facing the prospect of impaired vision or further sight reduction, our patients were highly anxious and experienced high levels of concern which were not 
always disclosed to staff. Medical and nursing staff need to anticipate and acknowledge the anxiety of these patients and provide support and reassurance.

Expert medical care was a necessity for these patients, which for some entailed travelling long distances. This is the reality for most specialist care for non-metropolitan patients in Australia due to the size of the continent and because most specialist services are based in metropolitan centres $(23,24)$. Strategies such as teleconferencing and outreach services $(25,26)$ have been implemented to overcome some of the access issues and should always be a consideration by policy planners in the provision of specialty health services.

Patients in unfamiliar environments and experiencing pain focus on the immediate concerns of relief of symptoms and treatment for their acute eye disease. Considerations such as the quality of medical or nursing care or the comfort of their environment, reported in other studies and situations (27), were not a priority in our study. In interviews the emphasis and concern was consistently primarily their vision and treatment. This could be due to the acute nature and unexpected occurrence that typifies microbial keratitis, and the unpredictable outcomes and response to treatment (2). The concern of patients for their vision and its treatment should be recognised by the healthcare team and measures implemented to reduce stress through clear communication, especially regular progress updates, education and support.

\section{Safe-guarding home and normal life}

Any person who requires acute hospital admission may face similar stresses regarding home life but very little Australian literature reports the patient experience as studies generally rely on surveys to measure patients' hospital experience which limits the capture of individual nuances (27). Again, the stress and anxiety expressed by patients can be alleviated by strategies to support them, such as clear communication and blanket review by support staff such as social workers. Access to family should be encouraged to limit feelings of isolation and concerns for both patients and carers.

Uncertainty regarding vision outcomes was a source of stress for all patients and family carers, whether related to continuing or future employment, driving or hobbies. Potential loss of employment and income is an easily recognizable cause for anxiety. Less easily recognised, perhaps, are the implications relating to uncertainly in the driving ability for the older population. To most older people driving is more than transportation and is also a symbol of freedom, continuing independence and self-reliance (28). Loss of ability to drive may therefore represent far more than a practical inconvenience and should be approached in this light when discussed with patients or their carers.

\section{Patients' vision-related quality of life}

Multiple versions of the NEIVFQ $(25,28,29$ and 51-item versions, with 3, 4 and 5-option responses) challenge direct comparisons. However, the NEIVFQ-25 has been used with other groups, allowing location of this sample in relation to clinical and community groups. Findings are available for microbial 
keratitis (3), cataract, glaucoma $(11)$ and macular degeneration $(11,29)$ samples; from dry eye syndrome out-patient and community samples $(30)$ and population reference groups $(11,29)$ including an older group (60 years and over) with normal eye health (31) (Table 3 ).

\section{(Table 3 about here)}

Broadly, of the patient groups, those with macular degeneration generally scored worse and the glaucoma group best, and all patient groups were outscored by the population and community groups. Our study sample generally scored similarly to the cataract and glaucoma groups and joined with the other keratitis and dry eye outpatient groups for top pain scores. This is perhaps not surprising as our sample was experiencing acute or acute on chronic disease sufficient to require hospitalisation. High levels of ocular pain have been reported in the initial phase of the disease (2), supporting our findings. For overall NEIVSQ-25, general health and driving scores our group scored better than most other patient groups; for general vision and vision-specific mental health, social functioning, role limitation and dependency, only patients with macular degeneration consistently fared worse.

The distribution of these NEIVFQ-25 scores across five clinical, population and community samples demonstrates the variability of symptom profiles within and between groups (Table 3 ), further complicated by differing demographic profiles. The other keratitis group was somewhat younger (mean 48 years) but reported substantial long-standing chronic disease (3) possibly responsible for this group's consistent low scores. Suzukamo et al's (2005) groups were mostly female and older than our cohort, as was the other macular degeneration group (mean 76.6 years; (29)). Participants with dry eye syndrome (30) were also mostly (81.8\%) female but markedly younger (mean 42.8 years), yet reported similar general health to our group.

Study limitations include that it was conducted at one site only, with a convenience sample of participants. Findings may not be representative, although we did reach data saturation, suggesting we had captured the range of potential experiences at least at this site.

\section{Conclusion}

In conclusion, this study highlights new knowledge regarding the experiences of patients and their carers admitted to a specialist eye service for the management of microbial keratitis. Clinicians should bear in mind the potentially devastating threat posed by microbial keratitis, exacerbated by its acute onset and the difficulty of planning or preparing for an emergency hospital admission. Healthcare providers need to be sensitive to the stresses experienced but not always voiced by these patients especially those related to possible vision loss and the impact of the diagnosis and hospital admission on home life and future lifestyle. Similar to other ocular diseases, these patients experience lower vision-related quality of life than population reference groups.

Study findings have messages for policy makers, clinicians, patients and families. Strategies should be developed to facilitate access to individualised care and support services, using multiple methods and 
modalities. The issue of the distances and methods for travel to a specialist eye service by patients suffering acute disease warrant consideration, with provision of transport support and affordable hostel accommodation for those not ready for discharge from the service but no longer in need of full hospital services. Provision of outreach services and service models including telehealth are also warranted for residents from distant areas. Discharge planning, particularly for patients travelling long distances, may be a challenge but early planning and clear communication with patients, family carers and those responsible for future review and follow up may improve this process. Given the high rates reported for complications and unplanned readmissions, service reconfiguration, enhanced discharge planning and post-discharge follow-up may be cost-effective as well as advantageous for patient and family wellbeing.

\section{Declarations}

\section{Ethics approval and consent to participate}

Approval to conduct the study was obtained from South Eastern Sydney Human Research Ethics Committee (reference: 151246; HREC/15/PoWH/491).

All participants provided informed consent to participate in the study.

\section{Consent for publication}

Written consent to publication was provided by all participants.

\section{Availability of data and material}

The datasets used and analysed during the current study are available from the corresponding author on reasonable request.

\section{Competing interests}

The authors declare that they have no competing interests

\section{Funding}

Funding was provided by the Save Sight Institute to fund the project research officer. Professor Watson is supported by a Sydney Medical School Foundation Fellowship. Dr Maria Cabrera Aguas is supported by the Sydney Eye Hospital Foundation. The funder played no part in the design of the study and collection, analysis, and interpretation of data or in writing the manuscript.

\section{Authors' contributions}

SW, MC-A and LP, with Agnes Shea, developed the proposal. S-A T collected the data, supported by MR and JM; S-AT, LP, MR and JM conducted analyses; S-AT and LP drafted the paper; all authors reviewed and contributed to revisions of the manuscript and approved the paper. 


\section{Acknowledgements}

The authors acknowledge the staff at the corneal unit and the hospital for their participation in the study. The author team acknowledge the contribution of Agnes Shea and Karen Tuqiri to the original development and funding of the study proposal. They thank the patients and family carers who participated in this study.

\section{References}

1. Collier S, Gronostaj M, MacGurn A, Cope J, Awsumb K, Yoder J, et al. Estimated burden of keratitis United States 2010. Morbidity and Mortality Weekly Report. 2014;63(45):1027-30.

2. Keay L, Edwards K, Naduvilath T, Taylor H, Snibson G, Forde K, et al. Microbial keratitis: predisposing factors and morbidity. Ophthalmology. 2006;113(1):109-16.

3. Li Y, Hong, J, Wei A, Wang X, Chen Y, Cui X, et al. Vision-related quality of life in patients with infectious keratitis. Optom Vis Sci. 2014;91(3):278-83.

4. Green M, Apel A, Stapleton F. Risk factors and causative organisms in microbial keratitis. Cornea. 2008;27(1):22-7.

5. Keay L, Edwards K, Maduvilath T, Forde K, Stapleton F. Factors affecting the morbidity of contact lens related microbial keratitis: a population study. Invest Ophthalmol Visual Sci. 2006;47:4302-8.

6. Taylor HR, Pezzullo ML, Keeffe JE. The economic impact and cost of visual impairment in Australia. $\mathrm{Br}$ J Ophthalmol. 2006;90(3):272-5.

7. Butler T, Spencer N, Chan C, Gilhotra J, McClellan K. Infective keratitis in older patients: a 4 year review, 1998-2002. Br J Ophthalmology. 2005;89:591-6.

8. Madden A, Simmons D, McCarty C, Khan M, Taylor H. Eye Health in Rural Australia. Clinical and Experimental Ophthalmology. 2002;30:316-2.

9. Ruckholdt M, Tofler G, Buckley T. The impact of hospitalisation on a visiting family member: a case study and discussion. Australian Critical Care. 2017;30(3):182-5.

10. Health Roundtable. Health Roundtable Report 2013-14: Eye Cornea Terrigal NSW: Health Roundtable; 2014 [

11. Suzukamo Y, Oshika T, Yuzawa M, Tokuda Y, Tomidokoro A, Oki A, et al. Psychometric properties of the 25-item National Eye Institute Visual Function Questionnaire (NEI VFQ-25), Japanese version. Health Quality Life Outcomes. 2005;3(65). 
12. Mangione C, Lee PP, Gutierrez P, Spritzer K, Berry S, Hays R. Development of the 25-item National Eye Institute Visual Function Questionnare (VFQ-25). Arch Ophthalmol. 2001;119:1050-8.

13. Creswell J, Piano V. Designing and Conducting Mixed Methods Research. California: Sage Publications; 2007.

14. Coulter A, Lancock L, Ziebland S, Calabresse J. Collecting data on patient experience is not enough: they must be used to improve care. BMJ 348g2225. 2014.

15. Parker R. Microbial Keratitis in inpatients at Sydney Eye Hospital in 2011. Sydney: University of Sydney; 2014.

16. Mangione C, Lee P, Pitts J, Gutierrez P, Berry S, Hays R. Psychometric properties of the National Eye Institute Visual Function Questionnaire (NEI-VFQ). Arch Ophthalmol. 1998;116(11):1496 - 504.

17. Braun V, Clarke V. Using thematic analysis in psychology. Qualitative Research in Psychology. 2006;3:77-101.

18. Morse J. Critical Analysis of Strategies for Determining Rigor in Qualitative Inquiry. Qualitative Health Research. 2015;25(9):1212-22.

19. National Eye Institute. 25 item Visual Function Questionnaire: version 20002000 [Available from: https://nei.nih.gov/catalog/visual-function-questionnaire-25.

20. Elmir R, Schmied V, Jackson D, Wilkes L. Interviewing people about potentially sensitve topics. Nurse Researcher. 2011;19(1):12-6.

21. Vision 2020. Eye Health Report Card: The state of Australia's eye health Melbourne2010 [Available from: http://www.vision2020australia.org.au/resources/eye-health-report-card-the-state-of-australias-eyehealth.

22. Boyle J, Vukicevic M, Koklanis K, Itsiopoulos C, Rees G. Experiences of patients undergoing repeated intravitreal ant-vascular endothelial growth factor injections for neurovascular aged related macular degeneration. Psychology, Health and Medicine. 2018;23(2):127-40.

23. Kelly J, Dwyer J, Willis E, Pekarsky B. Travelling to the city for hospital care: access facors in country Aboriginal patient journeys. Australian Journal of Rural Health. 2014;22:109-13.

24. McGrath P. Haematology patients" desire to access metropolitan hospital expertise. Australian Health Review. 2016;40:251-6.

25. Razavi H, Copeland SP, Turner AW. Increasing the impact of teleophthalmology in Australia: Analysis of structural and economic drivers in a state service. Australian Journal of Rural Health. 2016;25:45-52. 
26. Tuttle CSL, Carrington MJ, Stewart S, Brown A. Overcoming the tyrany of distance: An analysis of outreach visits to optimise secondary prevention of cardiovascular disease in high-risk individuals living in Central Australia. Australian Journal of Rural Health. 2015;24:99-105.

27. Harrison R, Walton M, Manias E, Mears S, Plumb J. Patients" experiences in Australian hospitals: a systematic review of evidence. Australian Health Review. 2017;41(419-435).

28. Oxley J, Charlton J. Attitudes to and mobility impacts of driving cessation. Topics in Geriatric Rehabilitation. 2009;25(1):43-54.

29. Chatziralli I, Mitropoulos P, Parikakis E, Niakas D, Labiris G. Risk factors for poor quality of life among patients with age-related macular degeneration. Seminars in Ophthalmology. 2017;32(6):772-80.

30. Le Q, Ge L, Li M, Wu L, Xu J, Hong J, et al. Comparison on the vision-related quality of life between outpatients and general population with dry eye syndrome. Acta Ophthalmologica. 2014;92:e124-e32.

31. Owsley C, McGwin G. Vision-targeted health related quality of life in older adults: patient -reported visibility problems in low luminance activities are more likely to decline than daytime activities BMC Ophthalmology. 2016;16(92).

\section{Table 3}

Due to technical limitations, Table 3 is only available as a download in the supplemental files section.

\section{Supplementary Files}

This is a list of supplementary files associated with this preprint. Click to download.

- supplement1.pdf 